\title{
The production of mature oocytes from adult ovaries following primary follicle culture in a marsupial
}

\author{
A Nation and L Selwood \\ Department of Zoology, The University of Melbourne, Parkville, Victoria 3010, Australia \\ Correspondence should be addressed to A Nation; Email: acnation@unimelb.edu.au
}

\begin{abstract}
A model marsupial culture system has been developed whereby individual primary follicles, obtained from adult ovaries, can be grown in vitro to the antral stage and oocytes retrieved from these follicles can achieve nuclear maturation (metaphase II) in the presence of LH. Primary follicles isolated from adult Sminthopsis macroura ovaries were cultured individually in one of four systems: microdrops under oil, upright, inverted, or roller culture. After 6 days of culture, cumulus-oocyte complexes (COCs) were excised from early antral follicles and incubated for an additional $24 \mathrm{~h}$ to assess meiotic competence and the effects of $\mathrm{LH}$ and lithium on oocyte maturation. Histology and transmission electron microscopy established normal in vivo standards and verified oocyte and follicular integrity following culture. On day 6 of culture, follicle viability was significantly greater in the inverted system $(73 \%)$ than in the other three systems $(10-46 \%)$. The inverted system was the most effective in supporting development with follicles demonstrating progressive growth during culture and showing antral signs by day 4 . Meiotic resumption during $\mathrm{COC}$ culture was facilitated by $\mathrm{LH}$, but hindered by lithium. The ability to resume meiosis and progress to metaphase II was equivalent in oocytes retrieved following follicle culture and those matured in vivo. This study highlights the importance of oxygen and nutrient availability during marsupial follicle culture, and demonstrates for the first time that primary follicles isolated from adult mammalian ovaries can undergo normal growth and development in vitro, to produce mature, meiotically competent oocytes.
\end{abstract}

Reproduction (2009) 138 247-255

\section{Introduction}

Assisted reproductive technologies (ART) rely upon the availability of mature, fertilizable oocytes. In the adult ovary, only a small number of oocytes are selected to develop each cycle. Superovulation is routinely used to increase oocyte yield; however, the number of in vivo matured oocytes available for ART purposes remains prohibitively low. Limited oocyte number, which compromises the use and success of IVF and ICSI, has, in recent years, prompted researchers to explore the possibility of maturing oocytes outside the ovary.

Follicle culture is an experimental technique that enables the predictable production of mature oocytes by utilizing the rich reserve of growing oocytes within the ovary. The ability to produce fully grown, developmentally competent oocytes in vitro from primary or primordial follicles would provide essential material for IVF and ICSI, and used in conjunction with tissue cryopreservation and embryonic transfer could provide new applications for fertility preservation in endangered wildlife species, economically important livestock, or humans undergoing cancer treatment.

To date, the most promising in vitro growth systems have been developed in mice, using newborn or prepubertal ovarian tissue. Specifically, oocytes from primordial and primary follicles can be grown to antral stages using microdrop culture (Eppig \& Schroeder 1989, Spears et al. 1994, Cortvrindt et al. 1996), and using either alginate hydrogel three-dimensional culture (Xu et al. 2006) or a two-step system comprising whole ovarian culture and cumulus-oocyte complex (COC) culture, live offspring have been produced (Eppig \& O'Brien 1996, O'Brien et al. 2003).

Among other eutherian species, culture systems, which enable the initiation of primordial follicle growth and development, have been described in humans (Hovatta et al. 1997, 1999, Wright et al. 1999), baboons (Wandji et al. 1997), sheep (Muruvi et al. 2005), and cattle (Braw-Tal \& Yossefi 1997). Systems that allow the development of antral follicles from secondary stage follicles have been reported in sheep (Cecconi et al. 1999, Newton et al. 1999), cattle (Gutierrez et al. 2000, McCaffery et al. 2000), and pigs (Hirao et al. 1994, Wu et al. 2001). While these achievements are remarkable, for follicle culture to be of benefit in the conservation of threatened species, or indeed the preservation of human fertility, we cannot rely upon the use of juvenile (neonatal or prepubertal) tissue.

Challenged by a limited source, culture systems that require immature ovaries have little practical application. A more realistic, functional approach is to 
develop a system that can support the growth of follicles from adult ovaries, enabling the utilization of tissue from infertile or recently deceased individuals. To date, the use of adult ovaries as a source of immature oocytes has been almost exclusively the domain of human and bovine research. Culture systems that support the initiation of follicle growth within ovarian cortical strips have been established (Hovatta et al. 1997, 1999, Wright et al. 1999), and isolated secondary follicles can mature in vitro to early antral stages (McCaffery et al. 2000, Thomas et al. 2001, Telfer et al. 2008). Nonetheless, progress towards developing a culture system that allows the complete growth of adult-derived primordial or primary follicles through antrum development to oocyte maturity remains a major challenge.

Here, we report the development of a model marsupial culture system, whereby individual, primary follicles from adult ovaries can be grown in vitro to antral stages, producing mature, meiotically competent oocytes.

\section{Results}

\section{Follicle classification}

In vivo matured follicles $(n=2812)$ were classified by morphology (Pedersen \& Peters 1968) and divided into stages (Table 1). Mean follicle diameter measurements revealed considerable size overlap between follicle categories (Table 1). Cultured follicles were subsequently assigned to stages on the basis of granulosa and theca layer descriptions, rather than size.

\section{Follicle growth}

Prior to culture (day 0 ), the mean diameter of selected primary follicles ranged from 63.6 to $215.5 \mu \mathrm{m}$, with an average ( \pm S.E.M.) of $163.2 \pm 1.6 \mu \mathrm{m}$. At the start of culture, there was no significant difference in mean follicle diameter between the four culture systems, $F_{(3,443)}=5.11, P>0.05$ (Table 2). During the first $24 \mathrm{~h}$ of culture (day 1), mean follicle diameter increased significantly in the upright, inverted, and roller systems, with follicles demonstrating greatest growth in the inverted system (Fig. 1). This trend continued throughout the 6-day culture period. Follicles cultured in the inverted system showed significant daily growth between days $1-3$ and days $4-5$, and subsequent stage progression (Table 1 ) reaching antral stages with a mean follicle diameter of $379.2 \pm 15.9 \mu \mathrm{m}$ by day 6 (Fig. 1 and Table 2). Following the first $24 \mathrm{~h}$, follicles in the upright and roller systems demonstrated progressive but nonsignificant growth, which appeared to plateau prior during the second half of the culture period (Fig. 1). The final mean diameter of follicles cultured in the upright $(205.8 \pm 13.9 \mu \mathrm{m})$ and roller $(264.8 \pm 14.4 \mu \mathrm{m})$ systems corresponds in vivo with the secondary and secondarytertiary transition stage respectively (Table 1 ). Both were significantly lower than the final mean diameter achieved in the inverted system (Table 2). The microdrop system was by far the least effective, with no significant change in mean follicular diameter throughout the culture period (Fig. 1 and Table 2).

\section{Follicle morphology}

Prior to culture, all primary follicles comprised a single layer of granulosa cells, surrounded by a few fibroblastlike theca cells and varying amounts of stromal tissue. Throughout the culture period, viable follicles maintained their follicular architecture, the oocyte remained relatively central, and the basement membrane appeared intact. The zona pellucida (ZP) initially appeared patchy, gradually developing a uniform appearance. The extent of antrum formation was used to categorize larger follicles into stages. The appearance

Table 1 Classification of Sminthopsis macroura ovarian follicles in vivo.

\begin{tabular}{|c|c|c|c|c|c|}
\hline \multirow[b]{2}{*}{ Stage } & \multirow[b]{2}{*}{ Type $^{a}$} & \multirow[b]{2}{*}{$n$} & \multicolumn{2}{|c|}{$\begin{array}{l}\text { Follicle diameter } \\
\qquad(\mu \mathrm{m})\end{array}$} & \multirow[b]{2}{*}{ Defining characteristics used for classification } \\
\hline & & & Min & Max & \\
\hline Primordial & 3а & 76 & 27 & 38 & One layer of squamous follicle cells surrounds the oocyte \\
\hline Primary & $3 b$ & 1108 & 36 & 213 & $\begin{array}{l}\text { One layer of cuboidal follicle cells (GCs) surrounds the growing oocyte. } \\
\text { The ZP appears patchy and irregular }\end{array}$ \\
\hline Secondary & 4 & 476 & 180 & 256 & $\begin{array}{l}\text { More than one layer of GCs surrounds the growing oocyte. The ZP appears } \\
\text { uniform. TCs begin to proliferate }\end{array}$ \\
\hline Transition to tertiary & 5 & 744 & 221 & 289 & Three or more GC layers surround the growing oocyte. TCs form layers \\
\hline Tertiary & 6 & 200 & 277 & 380 & $\begin{array}{l}\text { Distinct fluid-filled pockets appear among the GCs, which now comprise } \\
\text { many layers. TCs begin to differentiate (interna/externa) }\end{array}$ \\
\hline Antral & 7 & 208 & 301 & 576 & $\begin{array}{l}\text { Antral fluid continues to accumulate. Pockets merge into lacunae, } \\
\text { eventually coalescing to form a single antrum. The GCs differentiate } \\
\text { (cumulus/mural). TC differentiation continues }\end{array}$ \\
\hline Preovulatory & 8 & & & & \\
\hline
\end{tabular}

GC, granulosa cell; ZP, zona pellucida; TC, theca cell.

a Pedersen \& Peters (1968) classification. ${ }^{b}$ Mean follicle diameter was calculated at the largest follicle cross-section by measuring two perpendicular axes. Minimum and maximum mean diameters are given for each follicle stage. 
Table 2 Comparison of Sminthopsis macroura primary follicle culture systems.

\begin{tabular}{lllll}
\hline & Microdrop & Upright & Inverted & Roller \\
\hline Number of follicles $(n)$ & 107 & 120 & 152 & 68 \\
Initial mean diameter (day 0) \pm s.E.M. $(\mu \mathrm{m})$ & $166.9 \pm 10.1^{*}$ & $164.3 \pm 6.71^{*}$ & $162.3 \pm 5.9^{*}$ & $159.5 \pm 6.2^{*}$ \\
Final mean diameter (day 6) \pm s.E.M. $(\mu \mathrm{m})$ & $156.6 \pm 7.6^{*}$ & $205.8 \pm 13.9^{+}$ & $379.2 \pm 15.9^{\ddagger}$ & $264.8 \pm 14.4^{\S}$ \\
Most numerous follicle stage after culture (day 6) & Primary & Secondary & Antral & Secondary \\
Follicle quality & $10 \%$ & $32 \%$ & $73 \%$ & $46 \%$ \\
Viable & $90 \%$ & $68 \%$ & $27 \%$ & $54 \%$ \\
Degenerate & & & \\
\hline
\end{tabular}

Values with different superscripts differ significantly; $P<0.05$. There was a significant association between follicle quality and the culture system used, $\chi^{2}(3)=109.28, P<0.001$.

of small shadowy patches within the granulosa layer of cultured tertiary follicles, type 6 (Pedersen \& Peters 1968), indicated the beginning of antrum formation. As antral fluid accumulated, the patches coalesced to form lacunae, type 7 (Pedersen \& Peters 1968), and eventually a large single antrum, types 7 and 8 (Pedersen \& Peters 1968). Histological (Fig. 2A and B) and ultrastructural (Fig. 2C-H) examination further confirmed that follicles cultured in the inverted system were developing normally and conforming to in vivo standards (Kress et al. 2001). The oocyte appeared normal, with numerous microvilli extending towards the ZP (Fig. 2C). Outside the ZP, the granulosa cells (Fig. 2E) appeared healthy and were interspersed with antral pockets of varying size. The basement membrane remained intact, and by day 4 onwards the more advanced follicles showed differentiation of thecal cells into the steroidogenic theca interna and the more fibrous theca externa (Fig. 2G).

\section{Oocyte morphology}

Following 6 days of inverted follicle culture, 93\% (104 out of 111) of intact viable follicles had a diameter $>300 \mu \mathrm{m}$, and $83 \%$ (87 out of 104) of these yielded morphologically normal oocytes. Out of these, 90\% (78 out of 87 ) remained at the germinal vesicle (GV) stage, enclosed by intact cumulus cells (type 2 oocytes). The remaining $10 \%$ (9 out of 87 ) had shed most cumulus cells and undergone GV breakdown (GVBD) with extrusion of the first polar body to form type 3 oocytes. All follicles yielding type 3 oocytes had a diameter $>350 \mu \mathrm{m}$ (Fig. 3).

\section{COC culture}

Three-way log-linear analysis to test the relationship between oocyte origin, COC culture treatment, and oocyte maturation revealed that there was no significant association between the origin of oocytes and their ability to undergo meiotic resumption during COC culture, $\chi^{2}(3)=0.314, P=0.957 . \chi^{2}$-tests, performed separately for freshly isolated in vivo matured oocytes $\left(\chi^{2}(3)=32.75, \quad P<0.001\right)$ and oocytes retrieved following inverted follicle culture $\left(\chi^{2}(3)=21.37\right.$,
$P=0<0.001$ ), showed a significant relationship between culture treatment and oocyte maturation. In comparison with control data, the ability of type 2 oocytes to resume meiosis during $24 \mathrm{~h}$ COC culture was greatly improved by the addition of $\mathrm{LH}(10 \mu \mathrm{g} / \mathrm{ml})$. The presence of $\mathrm{LiCl}(10 \mathrm{mM} / \mathrm{ml})$ had little effect on oocyte maturation frequencies; however, $\mathrm{Li}_{2} \mathrm{CO}_{3}(10 \mathrm{mM} / \mathrm{ml})$ appeared to hinder development (Table 3 ).

\section{Discussion}

This is the first report in mammals of oocyte maturation in vitro using primary follicles sourced from adult ovaries, and one of the first descriptions of follicle culture in a marsupial. Using optimized culture conditions, this study has demonstrated that primary follicles harvested from adult ovarian tissue may be grown in vitro to antral stages.

The ability to culture follicles from adult ovaries and produce normal oocytes should prove instrumental in enhancing current reproductive technology, providing essential material for IVF and ICSI, and much needed insight into oocyte and follicle development.

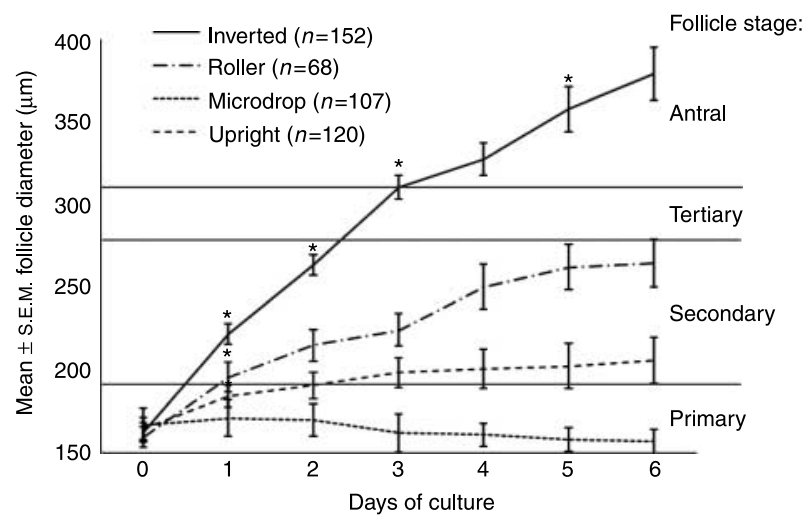

Figure $1 S$. macroura follicle growth in vitro showing the mean ( \pm s.E.M.) follicle diameter recorded during culture (days 0-6) in each of the four culture systems. The inverted culture system was most conducive to follicle growth. Primary follicles cultured in this system demonstrated progressive growth and stage progression throughout the culture period. *Indicates that the mean follicle diameter was significantly different to the previous day in the given culture system. The horizontal lines approximate transition between follicle stages (labelled on the right) based on measurements collected from in vivo samples (Table 1). 
Table 3 The influence of culture supplements on the meiotic ability of Sminthopsis macroura oocytes during $24 \mathrm{~h}$ COC culture. Oocyte frequencies were calculated separately for freshly isolated and cultured follicles.

\begin{tabular}{|c|c|c|c|c|c|c|}
\hline \multirow[b]{3}{*}{ Treatment } & \multicolumn{6}{|c|}{ The frequency (\%) of oocyte type after COC culture } \\
\hline & \multicolumn{3}{|c|}{ Freshly isolated follicles } & \multicolumn{3}{|c|}{ Cultured follicles } \\
\hline & $n$ & Type 2 & Type 3 & $n$ & Type 2 & Type 3 \\
\hline Control & 30 & 21.4 & 9.2 & 21 & 19.7 & 7.9 \\
\hline LH $(10 \mu \mathrm{g} / \mathrm{ml})$ & 30 & 5.1 & 25.5 & 23 & 7.9 & 22.4 \\
\hline $\mathrm{LiCl}(10 \mathrm{mM} / \mathrm{ml})$ & 18 & 13.3 & 5.1 & 17 & 17.1 & 5.3 \\
\hline $\mathrm{Li}_{2} \mathrm{CO}_{3}(10 \mathrm{mM} / \mathrm{ml})$ & 20 & 18.4 & 2.0 & 15 & 18.4 & 1.3 \\
\hline
\end{tabular}

The technique would provide greater options for the treatment of premature ovarian failure in humans and enable the propagation of important livestock and threatened wildlife species. The use of adult tissue is of particular interest to endangered species conservation as it would allow the collection of viable oocytes from recently deceased, adult, or infertile animals.

Despite recent eutherian advances (Eppig \& O'Brien 1996, Eppig et al. 1996, Hardy et al. 2000, Liu et al. 2002, Rizos et al. 2002, Carrell et al. 2005, Xu et al. 2006), reports of marsupial follicle culture remain scarce and success has been limited by inadequate growth rate and development. The first marsupial study, using preantral follicles from prepubertal ovaries of the grey short-tailed opossum, Monodelphis domestica, showed that individual preantral follicles cultured in microdrops grew to antral size without antrum formation, and determined the importance of FSH as a follicle growth stimulant in vitro (Butcher \& Ullmann 1996). Two additional studies have described marsupial follicle culture, both using follicles collected from adult ovaries and employing a similar microdrop system. In the tammar wallaby, Macropus eugenii, secondary follicles demonstrated significant growth over a 4-day culture period, but failed to progress to the tertiary follicle stage (Richings et al. 2006). In Sminthopsis macroura, early antral (lacunae stage) follicles showed follicle expansion in vitro during a 24-h culture period (Maleszewski \& Selwood 2004), and this work ascertained the requirement of exogenous LH for oocyte maturation in vitro.

The present study comprises the first complete (preantral-antral) description of normal growth and development of marsupial follicles in vitro. Having achieved maturation to metaphase II, the next obvious step is to assess the developmental competence of oocytes by examining rates of fertilization and embryonic development. Unfortunately, the techniques required for such analysis are not yet established in marsupials (Mate 1996, Magarey \& Mate 2003, Richings et al. 2004). To date, successful IVF has only been achieved in M. domestica (Moore \& Taggart 1993, Taggart et al. 1993) and ICSI in M. eugenii (Richings et al. 2004), neither study, however, attempted surrogate transfer to follow embryonic development to birth.
The use of a microdrop system to culture isolated follicles has proved relatively successful and adaptable across eutherian species; however, this study and previous work (Butcher \& Ullmann 1996, Richings et al. 2006) suggest that the technique provides inadequate support for the development of marsupial follicles in vitro. By comparing the microdrop culture system with three other systems specifically designed to increase nutrient and/or oxygen supply to the follicle, it became apparent that manipulation of these elements is critical to optimizing follicle culture success. Increasing the media volume and thus the availability of nutrients (upright, inverted and roller systems) resulted in a significant improvement in follicle growth and survival in all three systems. Similarly, by increasing oxygen availability (inverted and roller systems) follicle development was further enhanced. The inverted culture system proved by far the most superior, producing antral stage follicles equivalent both in size and morphology to their in vivo matured counterparts, suggesting that oxygen access, rather than nutrient supply, may be most crucial to follicle culture success.

The exact mechanism by which $\mathrm{LH}$ stimulates meiotic resumption (Channing et al. 1978) in arrested oocytes remains unknown, although there is growing evidence to support the involvement of phosphoinositide metabolism and intracellular calcium signaling (Eppig 1991, 1993, Homa et al. 1993, Coticchio \& Fleming 1998). In mice, the addition of $\mathrm{LiCl}$ to COC culture has generated contrasting results (Bagger et al. 1993, Pesty et al. 1994), leading to the hypothesis that spontaneous and hormone-induced meiotic resumption operate through different mechanisms (Coticchio \& Fleming 1998). In the present study, we found that lithium, a known inhibitor of phosphoinositide metabolism, suppressed oocyte maturation during COC culture by delaying GVBD. These results correlate with previous findings in mice (Pesty et al. 1994, Coticchio \& Fleming 1998), and suggest that activation of the phosphoinositide pathway, following the $\mathrm{LH}$ surge, is required for meiotic resumption in both eutherian and marsupial oocytes. This raises the possibility of using lithium as a mammalian fertility control agent. In accordance with previous work 

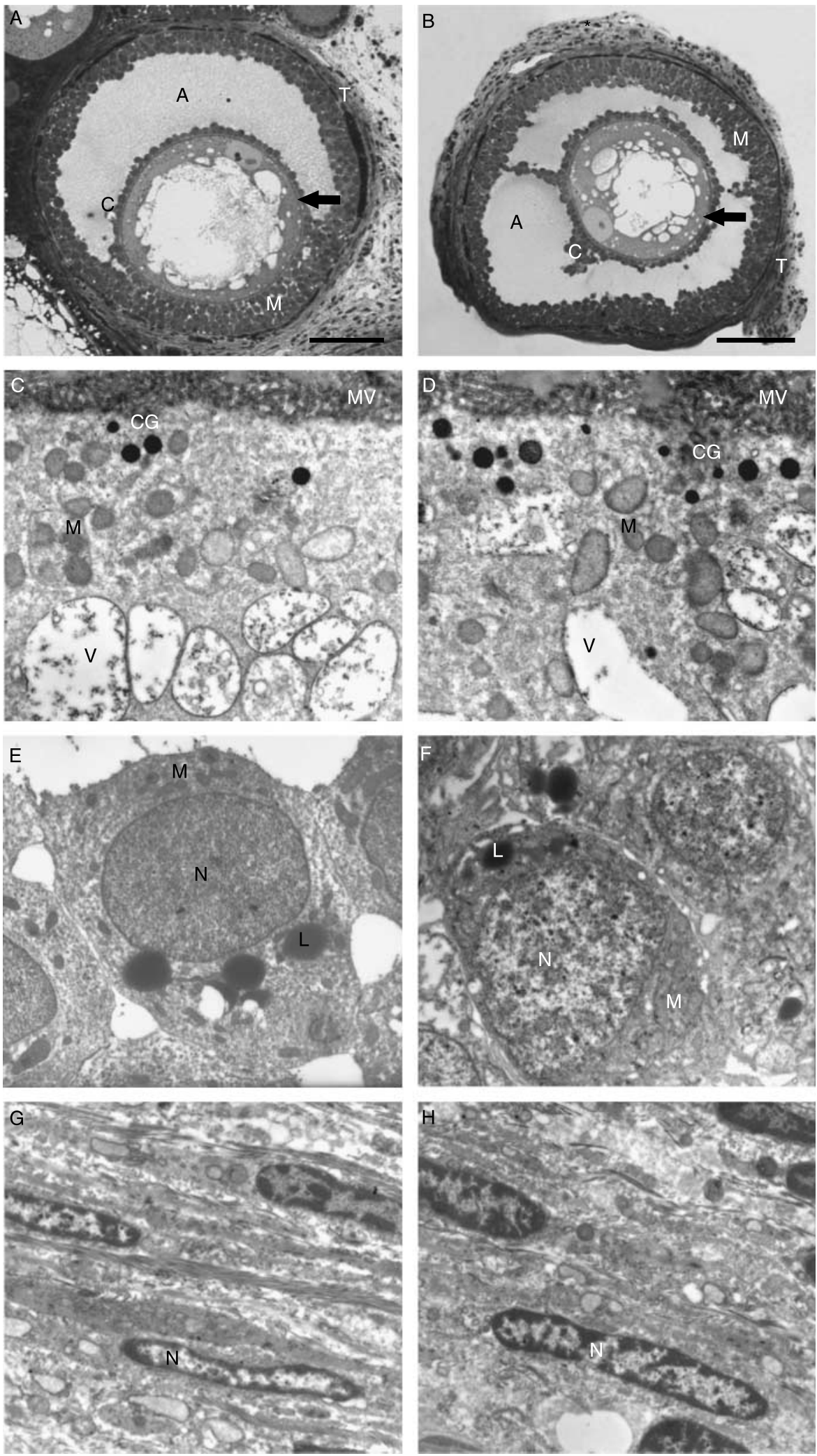

Figure 2 Histological and ultrastructural comparison of $S$. macroura antral stage follicles following maturation in vivo and in vitro revealed little difference. In addition to follicle growth data, this strongly suggests that primary follicles and their associated oocytes, cultured in the defined inverted system, grew and developed in a manner consistent with in vivo maturation. ( $\mathrm{A}$ and $\mathrm{B}$ ) Light micrographs of in vivo (A) and in vitro (B) matured antral stage follicles. Both follicles contain a large, spherical primary oocyte with a central concentration of empty-looking vesicles. The germinal vesicle (arrowhead) is situated in the peripheral, largely vesicle-free region of cytoplasm, which borders the zona pellucida (arrow). Outside the zona, a single layer of cumulus granulosa cells (C) surrounds the oocyte. The fluid-filled antrum (A) is well developed. Multiple layers of mural granulosa cells (M) separate the antrum from the basement membrane, which is surrounded by stromal theca cells $(\mathrm{T})(\mathrm{Scale}=100 \mu \mathrm{m})$. (C and D) Electron micrographs showing sections of antral follicle primary oocytes after maturation either in vivo (C) or in vitro (D). The ooplasm contains an abundance of mitochondria (M) and membrane-bound cytoplasmic vesicles (V). Cortical granules (CG) are predominately situated beneath the oocyte plasma membrane, and extensive microvilli (MV) processes extend into the zona pellucida (X 6610). (E and F) Electron micrographs of granulosa cells from in vivo $(\mathrm{E})$ and in vitro (F) matured antral follicles. The granulosa cell nuclei $(\mathrm{N})$ appear large and rounded. The most prominent cytoplasmic organelles are large lipid droplets $(\mathrm{L})$, containing stored cholesterol esters and numerous variably shaped mitochondria (M). Short microvillus (MV) projections of the granulosa cells link one cell to the next (X 5200). (G and H) Electron micrographs of thecal cells from in vivo $(\mathrm{G})$ and in vitro (H) matured antral follicles. Both theca cells and their nuclei $(\mathrm{N})$ are elongated. Electron density of the nuclei is varied and chromatin appears in clumps along the nuclear membrane (X 6610). 

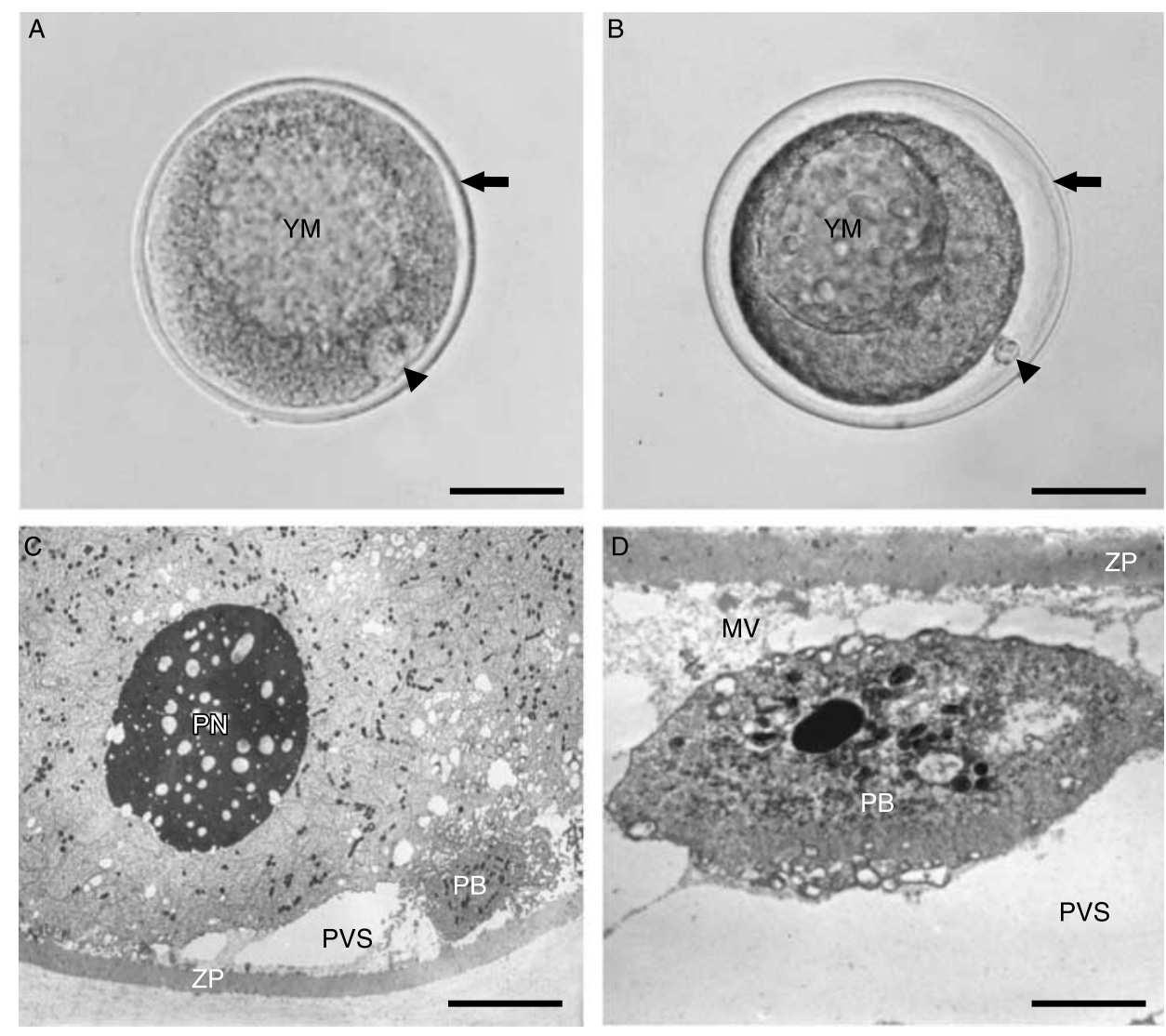

Figure 3 S. macroura oocyte maturation in vitro following follicle culture. (A) Light micrograph of a type 2 oocyte isolated from an early antral follicle on day 6 of follicle culture. The zona pellucida (arrow) is closely opposed to the oocyte and the germinal vesicle (arrowhead) is eccentric. The oocyte cytoplasm shows polarization with the presence of a yolk mass (YM) in the opposite hemisphere to the germinal vesicle. Cumulus granulosa cells have been removed by aspiration for greater photographic clarity (Scale $=80 \mu \mathrm{m}$ ). (B) Light micrograph of a type 3 oocyte (which has developed from a type 2 oocyte) after $24 \mathrm{~h} \mathrm{COC}$ culture in the presence of $10 \mu \mathrm{g} / \mathrm{ml} \mathrm{LH}$. A distinct perivitelline space has formed between the zona pellucida (arrow) and the oocyte plasma membrane, and within this the first polar body (arrowhead) may be seen. The yolk mass (YM) has concentrated and remains in the opposite hemisphere to the nuclear material (Scale $=80 \mu \mathrm{m}$ ). (C) Transmission electron micrograph of a type 3 oocyte, following both follicle culture and COC culture. Meiosis has resumed, one pronucleus (PN) has formed and the first polar body (PB) has been extruded into the perivitelline space (PVS). The female pronucleus is surrounded by a region of less dense, relatively organelle-free cytoplasm. Zona pellucida, ZP (X 1200). (D) Transmission electron micrograph of the first polar body (PB) extruded into the perivitelline space (PVS) during COC culture. Containing condensed chromatin, the polar body lies between the zona pellucida (ZP) and the oocyte plasma membrane (not shown), connected to both by microvilli (MV) (X 3900).

(Maleszewski \& Selwood 2004), our results also verify that $\mathrm{LH}$ influences meiotic resumption in marsupials as it does in eutherian mammals.

In conclusion, this novel study provides proof that adult ovaries may be used as a source of viable oocytes. With species-specific modifications, the inverted culture system reported here may be applied to other marsupial and/or eutherian species, thereby opening new avenues towards assisted reproduction and the conservation of endangered species.

\section{Materials and Methods}

\section{Chemicals}

Chemicals were purchased from Sigma-Aldrich unless otherwise specified.

\section{Animals}

S. macroura were obtained from a colony maintained by L Selwood at The University of Melbourne under Department of Sustainability and Environment permits. Colony maintenance and experiments followed Australian National Health and Medical Research Council Guidelines for the Care and Use of Animals for Scientific Purposes.

\section{Follicle isolation}

Mature females were killed by anesthetic overdose (Halothane; Rhone Meriux, West Footscray, VIC, Australia) followed by cervical dislocation. Blood collected from the posterior vena cava using a 21 gauge $(\mathrm{G})$ needle and $3 \mathrm{ml}$ syringe provided the serum required for each experiment. The reproductive tract was removed via a ventral midline incision and washed in calcium- and magnesium-free PBS at $35^{\circ} \mathrm{C}$, the basal body 
temperature of $S$. macroura. Under a laminar flow hood, the ovaries were removed and transferred immediately into $35^{\circ} \mathrm{C}$ HEPES-buffered DMEM containing $3 \mathrm{mg} / \mathrm{ml}$ BSA. Individual follicles were microdissected from the ovary at $\times 40$ magnification using $29 \mathrm{G}$ insulin syringes with the plungers removed.

\section{Follicle classification and morphology}

In vivo matured ovarian follicles $(n=2812)$ were serially sectioned and classified by morphological appearance (Pedersen \& Peters 1968), in order to establish standards for the evaluation of cultured follicles. Mean follicle diameter was calculated at the largest follicle cross-section by measuring two perpendicular axes, basement membrane to basement membrane, with a calibrated ocular micrometer. Follicles that presented pyknotic bodies in granulosa cells, low cellular density, a shrunken oocyte or condensed oocyte nucleus were considered atretic and not included in the analysis.

\section{Follicle culture}

Following ovarian microdissection, isolated preantral follicles were examined by confocal microscopy. Only follicles that were relatively translucent contained a single layer of cuboidal granulosa cells, Type 3b (Pedersen \& Peters 1968), a rounded central oocyte and an intact spherical structure were selected for culture. Follicles were cultured in DMEM with $4.5 \mathrm{~g} / \mathrm{l}$ glucose, $100 \mathrm{IU} / \mathrm{ml}$ penicillin, $50 \mu \mathrm{g} / \mathrm{ml}$ streptomycin, $100 \mu \mathrm{g} /$ $\mathrm{ml}$ glutamine, and $100 \mu \mathrm{g} / \mathrm{ml}$ kanamycin, DMEM ${ }^{+}$(Merry et al. 1995), with the addition of supplements shown to improve follicle development in culture: insulin-transferrinselenium (Cain et al. 1995; ITS-X; insulin, $10 \mu \mathrm{g} / \mathrm{ml}$; transferrin, $5.5 \mu \mathrm{g} / \mathrm{ml}$; selenium, $6.7 \mathrm{ng} / \mathrm{ml}$; Gibco-Invitrogen); $100 \mu \mathrm{g} / \mathrm{ml}$ L-ascorbic acid (Murray et al. 2001); $1.0 \mathrm{IU} / \mathrm{ml}$ recombinant human FSH (Izadyar et al. 1998; Gonal-F; donated by Melbourne IVF); and 5\% homologous serum (Nayudu \& Osborn 1992), obtained from the experimental animal and heat-inactivated before use. The optimal concentration of each supplement was determined by preliminary trials (data not shown). Follicles were cultured at $35^{\circ} \mathrm{C}$ for 6 days in one of four systems, described below.

(1) Microdrop culture under oil (Butcher \& Ullmann 1996): follicles $(n=107)$ were cultured individually in $30 \mu$ drops of medium in $35 \mathrm{~mm}$ Falcon Petri dishes (Becton Dickson, Knoxfield, VIC, Australia). The droplets were covered with sterile equilibrated mineral oil to prevent evaporation and fluctuations in $\mathrm{pH}$ and temperature. In systems 1-3, incubation occurred in a humidified gas environment of $5 \% \mathrm{CO}_{2}$ in air, and half the volume of media was replaced every $48 \mathrm{~h}$.

(2) Upright culture (Wycherley et al. 2004): to increase nutrient supply, follicles $(n=120)$ were cultured in a larger volume of media $(100 \mu \mathrm{l})$ in 96 -well round-bottomed suspension tissue culture plates (Sarstedt Ltd, Leicester, UK).

(3) Inverted culture (Wycherley et al. 2004): follicles $(n=152)$ were cultured in $100 \mu \mathrm{l}$ drops of medium in 96-well round-bottomed suspension tissue culture plates (as above); however, in this system, the plates were inverted prior to incubation. The medium was held in place by surface tension and the follicle dropped down to lie on the media/ gas interface, thereby maximizing oxygen access. This system was employed to increase both nutrient availability and oxygen supply to the follicle. Twelve different 96-well plates were tested in a series of experiments to determine the most conducive to this technique (data not shown).

(4) Roller culture: individual follicles $(n=68)$ were cultured in $300 \mu \mathrm{l}$ of medium in $2 \mathrm{ml}$ glass bottles. The medium was equilibrated with a gaseous mixture $\left(90 \% \mathrm{~N}_{2}: 5 \% \quad \mathrm{O}_{2}: 5 \%\right.$ $\mathrm{CO}_{2}$ ) previously used in embryo culture (Eppig \& Schroeder 1989). Following gas infusion, the bottles were sealed and rotated continuously on rollers within an incubator. The constant movement within the bottle should facilitate gas and nutrient exchange between follicle and medium and also increase oxygenation of the medium by the surrounding gas. Half of the medium was replaced on day 3 , and the bottles re-gased.

In vitro matured follicles $(n=347)$ were examined prior to culture (day 0 ) and then every $24 \mathrm{~h}$ using an inverted microscope (Wild Leitz, Melbourne, VIC, Australia) with heated stage. Mean follicle diameter was calculated as previously described. Follicular development and viability were evaluated daily by assessing general morphology and structure, including ZP deposition, antral fluid accumulation, and theca development. Follicles with clear granulosa cells and an intact follicular structure were deemed viable, regardless of growth rate. Follicles assigned to the non-viable (atretic) category demonstrated one or more of the following characteristics: an absence of growth; an irregularly shaped, retracted, or darkening oocyte; dark, granular (apoptotic) granulosa cells; a damaged (thinning/budding) or ruptured thecal layer.

\section{Oocyte morphology}

Following 6 days of inverted follicle culture, viable follicles with a diameter $>300 \mu \mathrm{m}$ were carefully ruptured using $29 \mathrm{G}$ needles and oocytes or COCs collected. Oocyte maturity was assessed in terms of follicle type, nuclear content, and cytoplasmic polarity, according to Merry et al. (1995). Type 2 oocytes (Merry et al. 1995), recovered from early antral follicles, remained in meiotic arrest at the GV stage, contained centrally concentrated cytoplasmic vesicles and adherent cumulus cells. Type 3 oocytes (Merry et al. 1995), obtained from late antral follicles, were cumulus free, showed marked cytoplasmic polarity, and had undergone GVBD followed by extrusion of the first polar body.

\section{Oocyte maturation}

The ability of type 2 oocytes to resume meiosis and progress to metaphase II in vitro was compared between freshly isolated, in vivo matured oocytes and those retrieved following follicle culture. Type 2 oocytes and surrounding cumulus cells were transferred to $30 \mu \mathrm{l}$ drops of the above follicle culture medium supplemented with agents known to affect oocyte maturation: 
either $10 \mu \mathrm{g} / \mathrm{ml}$ porcine pituitary LH (Maleszewski \& Selwood 2004), a gift from Vetrepharm (London, Ontario, Canada); $10 \mathrm{mM} / \mathrm{ml} \mathrm{LiCl}$ (Bagger et al. 1993); or $10 \mathrm{mM} / \mathrm{ml} \mathrm{Li}_{2} \mathrm{CO}_{3}$. COCs were incubated at $35{ }^{\circ} \mathrm{C}$ in $6 \% \mathrm{CO}_{2}$ in air for $24 \mathrm{~h}$ in the previously described microdrop system. After $24 \mathrm{~h}$, oocyte maturity was assessed in terms of chromatin configuration, with oocytes graded as either type 2 (GV stage) or type 3 (Telophase/MII).

\section{Histological and ultrastructural analysis}

Isolated, non-cultured, and non-atretic follicles at all stages of folliculogenesis were collected to establish in vivo standards. Cultured follicles were randomly selected throughout the culture period and at its conclusion to validate in vitro observations and determine culture standards. Selected follicles were immersed in Superfix $(2.5 \%$ glutaraldehyde (ProSciTech, Thuringowa, QLD, Australia), 3\% paraformaldehyde (Merck-BDM), and $0.2 \mathrm{M}$ sodium cacodylate buffer) at room temperature (RT) for $2 \mathrm{~h}(\mathrm{pH} 7.4)$. After three rinses in $0.1 \mathrm{M}$ sodium cacodylate buffer $(\mathrm{pH} 7.4)$, post-fixation took place in $1 \%$ osmium tetroxide (ProSciTech) diluted with $0.2 \mathrm{M}$ sodium cacodylate buffer for $2 \mathrm{~h}$ at RT. Following rinsing in $0.1 \mathrm{M}$ sodium cacodylate buffer, the tissue was dehydrated in an ethanol series, infiltrated with a propylene oxide - EponAraldite mixture, embedded in pure Epon-Araldite and polymerized at $60{ }^{\circ} \mathrm{C}$ for $48 \mathrm{~h}$. The resin consisted of a mixture of $25 \mathrm{ml}$ Procure 812, $15 \mathrm{ml}$ Araldite 502, $55 \mathrm{ml}$ dodecenyl succinic anhydride, and $1.25 \mathrm{ml}$ benzyldimethylamine (ProSciTech). Semi-thin sections $(1 \mu \mathrm{m})$ were cut with glass knives on an ultramicrotome (Ultracut E; Reichert-Jung, Heidelberg, Germany) and stained with $1 \%$ toluidine blue and $1 \%$ sodium borate in distilled water at $90{ }^{\circ} \mathrm{C}$ for $1-2 \mathrm{~min}$. Thin sections $(70 \mathrm{~nm})$, cut with a diamond knife, were stained with $3 \%$ uranyl acetate and $0.6 \%$ lead citrate, mounted on coated grids, and examined with a transmission electron microscope (CM 10; Philips, Eindhoven, The Netherlands).

\section{Statistical analysis}

Mean follicle diameter on day 0 was compared between culture systems by one-way independent ANOVA followed by Bonferroni's post hoc correction. Follicle growth during culture was analyzed within each system by one-way repeatedmeasures ANOVA and Bonferroni's post hoc test. $\chi^{2}$-tests were used to compare follicle quality between culture systems and oocyte maturation in the presence or absence of supplements. Three-way log-linear analysis was employed to test the relationship between oocyte origin, the effect of culture supplements, and oocyte development. Differences were considered statistically significant when $P<0.05$. Data analysis was performed using SPSS 14.0 (Chicago, IL, USA).

\section{Declaration of interest}

The authors declare that there is no conflict of interest that could be perceived as prejudicing the impartiality of this research.

\section{Funding}

This work was supported by the Foundation for Research Science and Technology, New Zealand (MELB0301) and The University of Melbourne, Australia.

\section{Acknowledgements}

Much gratitude is due to Joan Clark for assistance with electron micrography.

\section{References}

Bagger P, Byskov A, Christiansen M, Bang L \& Mortensen L 1993 Lithium stimulates the first meiotic division in mouse oocytes. Acta Obstetricia et Gynecologica Scandinavica 72 514-519.

Braw-Tal R \& Yossefi S 1997 Studies in vivo and in vitro on the initiation of follicle growth in the bovine ovary. Journal of Reproduction and Fertility 109 165-171.

Butcher L \& Ullmann S 1996 Culture of preantral ovarian follicles in the grey, short-tailed opossum, Monodelphis domestica. Reproduction, Fertility, and Development 8 535-539.

Cain L, Chatterjee S \& Collings T 1995 In vitro folliculogenesis of rat preantral follicles. Endocrinology 136 3369-3377.

Carrell D, Liu L, Huang I \& Peterson C 2005 Comparison of maturation, meiotic competence, and chromosome aneuploidy of oocytes derived from two protocols for in vitro culture of mouse secondary follicles. Journal of Assisted Reproduction and Genetics 22 347-354.

Cecconi S, Barboni B, Coccia M \& Mattioli M 1999 In vitro development of sheep preantral follicles. Biology of Reproduction 60 594-601.

Channing C, Hillensjo T \& Schaerf F 1978 Hormonal control of oocyte meiosis, ovulation and luteinization in mammals. Clinics in Endocrinology and Metabolism 7 601-624.

Cortvrindt R, Smitz J \& Van Steirteghem A 1996 In-vitro maturation, fertilization and embryo development of immature oocytes from early preantral follicles from prepubertal mice in a simplified culture system. Human Reproduction 11 2656-2666.

Coticchio G \& Fleming S 1998 Inhibition of phosphoinositide metabolism or chelation of intracellular calcium blocks FSH-induced but not spontaneous meiotic resumption in mouse oocytes. Developmental Biology 203 201-209.

Eppig J 1991 Intercommunication between mammalian oocytes and companion somatic cells. BioEssays 13 569-574.

Eppig J 1993 Regulation of mammalian oocyte maturation. In The Ovary, pp 185-208. Eds E Adashi \& P Leung. New York: Raven Press.

Eppig J \& O'Brien M 1996 Development in vitro of mouse oocytes from primordial follicles. Biology of Reproduction 54 197-207.

Eppig J \& Schroeder A 1989 Capacity of mouse oocytes from preantral follicles to undergo embryogenesis and development to live young after growth, maturation and fertilization in vitro. Biology of Reproduction 41 268-276.

Eppig J, O'Brien M \& Wigglesworth K 1996 Mammalian oocyte growth and development in vitro. Molecular Reproduction and Development $\mathbf{4 4}$ 260-273.

Gutierrez C, Ralph J, Telfer E, Wilmut I \& Webb R 2000 Growth and antrum formation of bovine preantral follicles in long-term culture in vitro. Biology of Reproduction 62 1322-1328.

Hardy K, Wright C, Franks S \& Winston R 2000 In vitro maturation of oocytes. British Medical Bulletin $\mathbf{5 6} 588-602$.

Hirao Y, Nagai T, Kubo M, Miyano T, Miyake M \& Kato S 1994 In vitro growth and maturation of pig oocytes. Journal of Reproduction and Fertility 100 333-339.

Homa S, Carroll J \& Swann K 1993 The role of calcium in mammalian oocyte maturation and egg activation. Human Reproduction $\mathbf{8}$ 1274-1281.

Hovatta O, Silye R, Abir R, Krausz T \& Winston R 1997 Extracellular matrix improves survival of both stored and fresh human primordial and primary ovarian follicles in long-term culture. Human Reproduction 12 1032-1036. 
Hovatta O, Wright C, Krausz T, Hardy K \& Winston R 1999 Human primordial, primary and secondary ovarian follicles in long-term culture: effect of partial isolation. Human Reproduction 14 2519-2524.

Izadyar F, Zeinstra E \& Bevers M 1998 Follicle-stimulating hormone and growth hormone act differently on nuclear maturation while both enhance developmental competence of in vitro matured bovine oocytes. Molecular Reproduction and Development 51 339-345.

Kress A, Merry N \& Selwood L 2001 Oogenesis in the marsupial stripefaced dunnart, Sminthopsis macroura. Cells Tissues Organs $\mathbf{1 6 8}$ 188-202.

Liu J, Rybouchkin A, Van der Elst J \& Dhont M 2002 Fertilization of mouse oocytes from in vitro-matured preantral follicles using classical in vitro fertilization or intracytoplasmic sperm injection. Biology of Reproduction 67 575-579.

Magarey G \& Mate K 2003 Fertilization following intracytoplasmic sperm injection of in vivo and in vitro matured oocytes from an Australian marsupial, the tammar wallaby (Macropus eugenii). Zygote 11 339-346.

Maleszewski M \& Selwood L 2004 Induced parthenogenetic activation of oocytes of the marsupial Sminthopsis macroura. Reproduction, Fertility, and Development 15 559-604.

Mate K 1996 Cytoplasmic maturation of the marsupial oocyte during the periovulatory period. Reproduction, Fertility, and Development 8 509-519.

McCaffery F, Leask R, Riley S \& Telfer E 2000 Culture of bovine preantral follicles in a serum-free system: markers for assessment of growth and development. Biology of Reproduction 63 267-273.

Merry N, Johnson M, Gehring C \& Selwood L 1995 Cytoskeletal organization in the oocyte, zygote, and early cleaving embryo of the stripe-faced dunnart (Sminthopsis macroura). Molecular Reproduction and Development 41 212-224.

Moore H \& Taggart D 1993 In vitro fertilization and embryo culture in the grey short-tailed opossum, Monodelphis domestica. Journal of Reproduction and Fertility 98 267-274.

Murray A, Molinek M, Baker S, Kojima F, Smith M, Hillier S \& Spears N 2001 Role of ascorbic acid in promoting follicle integrity and survival in intact mouse ovarian follicles in vitro. Reproduction 121 89-96.

Muruvi W, Picton H, Rodway R \& Joyce I 2005 In vitro growth of oocytes from primordial follicles isolated from frozen-thawed lamb ovaries. Theriogenology 64 1357-1370.

Nayudu P \& Osborn S 1992 Factors influencing the rate of preantral and antral growth of mouse ovarian follicles in vitro. Journal of Reproduction and Fertility 95 349-362.

Newton H, Picton H \& Gosden R 1999 In vitro growth of oocyte-granulosa cell complexes isolated from cryopreserved ovine tissue. Journal of Reproduction and Fertility 115 141-150.

O'Brien M, Pendola J \& Eppig J 2003 A revised protocol for in vitro development of mouse oocytes from primordial follicles dramatically improves their developmental competence. Biology of Reproduction 68 1682-1686.
Pedersen T \& Peters H 1968 Proposal for a classification of oocytes and follicles in the mouse ovary. Journal of Reproduction and Fertility 17 555-557.

Pesty A, Lefevre B, Kubiak J, Geraud G, Tesarik J \& Maro B 1994 Mouse oocyte maturation is affected by lithium via the polyphosphoinositide metabolism and the microtubule network. Molecular Reproduction and Development 38 187-199.

Richings N, Shaw G, Temple-Smith P \& Renfree M 2004 Intra-cytoplasmic sperm injection in a marsupial. Reproduction 128 595-605.

Richings N, Shaw G, Temple-Smith P \& Renfree M 2006 Growth and histology of ovarian follicles after cold storage in the tammar wallaby. Reproduction, Fertility, and Development 18 677-688.

Rizos D, Ward F, Duffy P, Boland M \& Lonergan P 2002 Consequences of bovine oocyte maturation, fertilization or early embryo development in vitro versus in vivo: implications for blastocyst yield and blastocyst quality. Molecular Reproduction and Development 61 234-248.

Spears N, Boland N, Murray A \& Gosden R 1994 Mouse oocytes derived from in vitro grown primary follicles are fertile. Human Reproduction $\mathbf{9}$ 527-532.

Taggart D, O'Brien H \& Moore H 1993 Ultrastructural characteristics of in vivo and in vitro fertilization in the grey short-tailed opossum, Monodelphis domestica. Anatomical Record 237 21-37.

Telfer E, McLaughlin M, Ding C \& Thong K 2008 A two-step serum-free culture system supports development of human oocytes from primordial follicles in the presence of activin. Human Reproduction 23 1151-1158.

Thomas F, Leask R, Srsen V, Riley S, Spears N \& Telfer E 2001 Effect of ascorbic acid on health and morphology of bovine preantral follicles during long-term culture. Reproduction 122 487-495.

Wandji S, Srsen V, Nathanielsz P, Eppig J \& Fortune J 1997 Initiation of growth of baboon primordial follicles in vitro. Human Reproduction $\mathbf{1 2}$ 1993-2001.

Wright C, Hovatta O, Margara R, Trew G, Winston R, Franks S \& Hardy K 1999 Effects of follicle-stimulating hormone and serum substitution on the in-vitro growth of human ovarian follicles. Human Reproduction 14 1555-1562.

Wu J, Emery B \& Carrell D 2001 In vitro growth, maturation, fertilization, and embryonic development of oocytes from porcine preantral follicles. Biology of Reproduction 64 375-381.

Wycherley G, Downey D, MT K \& Hynes A 2004 A novel follicle culture system markedly increases follicle volume, cell number and oestradiol secretion. Reproduction 127 669-677.

Xu M, Kreeger P, Shea L \& Woodruff T 2006 Tissue-engineered follicles produce live, fertile offspring. Tissue Engineering 12 2739-2746.

Received 28 January 2009

First decision 2 March 2009

Revised manuscript received 16 April 2009

Accepted 2 June 2009 